\title{
Correction to: ASNC/AHA/ASE/EANM/HFSA/ ISA/SCMR/SNMMI expert consensus recommendations for multimodality imaging in cardiac amyloidosis: Part 1 of 2-evidence base and standardized methods of imaging
}

Sharmila Dorbala, MD, MPH, FASNC (Chair), ${ }^{a}$ Yukio Ando, MD, PhD, ${ }^{b}$ Sabahat Bokhari, $M D,{ }^{c}$ Angela Dispenzieri, $M D,{ }^{d}$ Rodney H. Falk, $M D,{ }^{a}$ Victor A. Ferrari, MD, ${ }^{\mathrm{e}}$ Marianna Fontana, PhD, ${ }^{\mathrm{f}}$ Olivier Gheysens, MD, PhD, ${ }^{\mathrm{g}}$ Julian D. Gillmore, MD, PhD, ${ }^{f}$ Andor W. J. M. Glaudemans, MD, PhD, ${ }^{\text {h }}$ Mazen A. Hanna, MD, ${ }^{i}$ Bouke P. C. Hazenberg, MD, PhD, ${ }^{j}$ Arnt V. Kristen, MD, ${ }^{k}$ Raymond Y. Kwong, MD, MPH, ${ }^{\mathrm{a}}$ Mathew S. Maurer, MD, ${ }^{\mathrm{c}}$ Giampaolo Merlini, MD, ${ }^{\mathrm{l}, \mathrm{m}}$ Edward J. Miller, MD, PhD, ${ }^{\mathrm{n}}$ James C. Moon, MD, ${ }^{\mathrm{f}}$ Venkatesh L. Murthy, MD, PhD, ${ }^{\circ}$ C. Cristina Quarta, MD, PhD, ${ }^{f}$ Claudio Rapezzi, MD, ${ }^{p}$ Frederick L. Ruberg, MD, ${ }^{\mathrm{q}}$ Sanjiv J. Shah, MD, ${ }^{\mathrm{r}}$ Riemer H. J. A. Slart, MD, ${ }^{\mathrm{h}}$ Hein J. Verberne, MD, PhD, ${ }^{\mathrm{s}}$ and Jamieson M. Bourque, MD, MHS, FASNC (Co-Chair) $^{\mathrm{t}}$

a Cardiac Amyloidosis Program, Cardiovascular Imaging Program, Departments of Radiology and Medicine, Harvard Medical School, Brigham and Women's Hospital, Boston, MA

b Department of Neurology, Graduate School of Medical Sciences, Kumamoto University, Kumamoto, Japan

c Columbia University Medical Center/New York Presbyterian Hospital, Columbia University, New York, NY

d Division of Hematology, Division of Cardiovascular Diseases, and Department of Radiology, Division of Nuclear Medicine, Department of Medicine, Mayo Clinic, Rochester, MN

e Perelman School of Medicine, University of Pennsylvania, Philadelphia, PA

f Division of Medicine, National Amyloidosis Centre, University College London, London, UK

g Nuclear Medicine and Molecular Imaging, University Hospitals Leuven, Leuven, Belgium

h Department of Nuclear Medicine and Molecular Imaging, Medical Imaging Center, University Medical Center Groningen, University of Groningen, Groningen, The Netherlands

i Department of Cardiovascular Medicine, Cleveland Clinic, Cleveland, $\mathrm{OH}$

j Department of Rheumatology \& Clinical Immunology, University Medical Center Groningen, University of Groningen, Groningen, The Netherlands

k Department of Cardiology, University of Heidelberg, Heidelberg, Germany

I Amyloidosis Research and Treatment Center, Foundation Istituto di Ricovero e Cura a Carattere Scientifico Policlinico San Matteo, Pavia, Italy

m Department of Molecular Medicine, University of Pavia, Pavia, Italy

n Cardiovascular Medicine, Yale University School of Medicine, New Haven, CT

- Frankel Cardiovascular Center, Michigan Medicine, Ann Arbor, MI

p Cardiology Unit, Department of Experimental, Diagnostic and Specialty Medicine, Alma MaterUniversity of Bologna, Bologna, Italy

The original article can be found online at https://doi.org/10.1007/ s12350-019-01760-6.

Reprint requests: Sharmila Dorbala, MD, MPH, FASNC (Chair), Cardiac Amyloidosis Program, Cardiovascular Imaging Program, Departments of Radiology and Medicine, Harvard Medical School,
Brigham and Women's Hospital, Boston, MA ; sdorbala@bwh. harvard.edu

J Nucl Cardiol 2021;28:1761-2.

$1071-3581 / \$ 34.00$

Copyright (C) 2021 American Society of Nuclear Cardiology. 
q Amyloidosis Center and Section of Cardiovascular Medicine, Department of Medicine, Boston University School of Medicine, Boston Medical Center, Boston, MA

$r$ Feinberg School of Medicine, Northwestern University, Chicago, IL

$s$ Department of Radiology and Nuclear Medicine, Amsterdam UMC, University of Amsterdam, Amsterdam, The Netherlands

t Departments of Medicine and Radiology, Cardiovascular Imaging Center, University of Virginia, Charlottesville, VA

doi: $10.1007 / \mathrm{s} 12350-021-02711-\mathrm{w}$

\section{CORRECTION TO:} https://doi.org/10.1007/s12350-019-01760-6

- In the Introduction SCMR was listed incorrectly. SCMR is the Society for Cardiovascular Magnetic Resonance.

- Figure 1 erroneously printed without Yen sign (¥) in 'Final Diagnosis.' Please see revised Figure 1.

- Acknowledgments erroneously printed without reviewers Richard Cheng, MD and Roy John, MD.

Publisher's Note Springer Nature remains neutral with regard to jurisdictional claims in published maps and institutional affiliations.

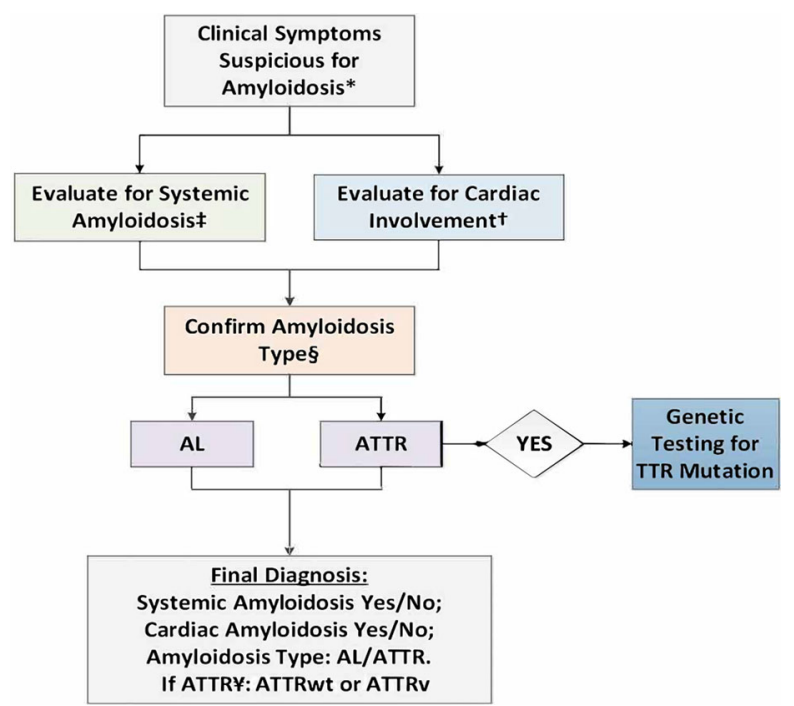

Figure 1. Systematic evaluation of cardiac amyloidosis. A comprehensive evaluation of cardiac amyloidosis includes consideration of clinical symptoms, evaluation of cardiac involvement (biomarkers and cardiac imaging), evaluation of systemic amyloidosis (serum, urine testing, and biopsy), followed by typing of amyloid deposits into AL or ATTR, and documentation of mutations in patients with ATTR amyloidosis. *Clinical symptoms: heart failure, peripheral/ autonomic neuropathy, macroglossia, carpal tunnel syndrome, periorbital bruising, stroke, atrial fibrillation, postural hypotension, fatigue, weight loss, pedal edema, renal dysfunction, diarrhea, constipation. ${ }^{\dagger}$ Evaluation for cardiac amyloidosis: ECG, ECHO, CMR, EMB, ${ }^{99 \mathrm{~m}} \mathrm{Tc}-\mathrm{PYP} / \mathrm{DPD} / \mathrm{HMDP} /{ }^{123} \mathrm{I}-$ $m$ IBG/PET, NT-proBNP, troponin T. ${ }^{+}$Evaluation for systemic amyloidosis: AL: detect plasma cell clone: serum and urine immunofixation, serum FLC assay and immunoglobulin analysis; AL: detect systemic organ involvement: 24-hour urine protein, alkaline phosphatase, eGFR, cardiac biomarkers (NT-proBNP, troponins); Tissue biopsy: EMB/Fatpad/Bone marrow/Other with Congo red staining. ${ }^{\S}$ Confirm Amyloidosis Type: ATTR: IHC and MS of Biopsy or ${ }^{99 \mathrm{~m}} \mathrm{Tc}-\mathrm{PYP} / \mathrm{DPD} /$ HMDP Grade 2 or3 if a clonal process is excluded; AL: MS or IHC of Biopsy. ${ }^{\ddagger}$ Confirm TTR mutation in patients with ATTR amyloidosis: genetic testing for TTR mutations. $A L$, amyloid light chain; ATTR, amyloid transthyretin; $C M R$, cardiac magnetic resonance imaging; $D P D,-3,3$-diphosphono-1,2-propanodicarboxylic acid; $E C G$, electrocardiogram; $E M B$, endomyocardial biopsy; $E C H O$, echocardiogram; $e G F R$, estimated glomerular filtration rate; $H M D P$, hydroxymethylenediphosphonate; $I H C, \quad$ immunohistochemistry; $m I B G$, meta-iodobenzylguanidine; $M S$, mass spectroscopy; $v$, hereditary; PYP, pyrophosphate; $T c$, technetium; wt, wild-type. 